\title{
Detection and characterization of polioviruses originating from urban sewage in Yaounde and Douala, Cameroon 2016-2017
}

Daniel Kamga Njile', Serge Alain Sadeuh-Mba', Marie-Claire Endegue-Zanga', Marcellin Nimpa Mengouo², Marlise Dontsop Djoumetio ${ }^{2}$, Franky Baonga Ba Pouth ${ }^{3}$, Ousmane Madiagne Diop ${ }^{4}$ and Richard Njouom ${ }^{1 *}$ (D)

\begin{abstract}
Objective: Transmission of wild polioviruses (WPVs) and vaccine-derived polioviruses (VDPVs) have been interrupted in Cameroon since July 2014. Subsequently, Cameroon withdrew Sabin type 2 from routine immunization in April 2016. This study aimed to investigate the detection rates and overtime distribution of the types of PVs recovered from urban sewage in Cameroon.

Results: From January 2016 to December 2017, 517 sewage specimens originating from Yaounde (325 specimens) and Douala (192 specimens) were analyzed. No WPVs and VDPVs were isolated in this study. In contrast, vaccine strains of poliovirus were detected throughout the study period. Isolates Sabin types 1 and 3 were sporadically detected whereas Sabin 2 was found only from January to May 2016 both in Yaounde and Douala. The absence of Sabin 2 in sewage specimens since June 2016 indicates its rapid disappearance after withdrawal from routine immunization in April 2016. This study provides substantial support to the observation that WPV and VDPVs have been successfully eliminated in Cameroon. However, it remains essential to maintain and extend high quality environmental surveillance as long as WPV reservoirs and VDPV outbreaks are detected in Africa.
\end{abstract}

Keywords: Poliovirus, Vaccine, Surveillance, Eradication, Sewage, Cameroon

\section{Introduction}

Enteroviruses (EVs) are ubiquitous human pathogens belonging to the genus Enterovirus and the family Picornaviridae. The genus Enterovirus include Non-Polio EVs (NPEVs) that infect both humans and animals as well as Polioviruses (PVs) that specifically infect humans [1, 2]. There is three serotypes of PVs (PV1, PV2, PV3) and each type is further divided into three categories based on the extent of nucleotide sequence divergence of their VP1 capsid coding gene compared to that of corresponding oral poliovirus vaccine (OPV) strains: (i) OPV-like or Sabin viruses $(<1 \%$ divergent for types 1 and 3 , and $<0.6 \%$ for type 2), (ii) Vaccine-derived PVs [VDPVs] (1-15\%

\footnotetext{
*Correspondence: njouom@pasteur-yaounde.org; njouom@yahoo.com

${ }^{1}$ Virology Service, National Reference and Public Health Laboratory,

Centre Pasteur of Cameroon, 451 Rue 2005, PO box 1274, Yaounde,

Cameroon

Full list of author information is available at the end of the article
}

divergent for PV1 and PV3 and 0, 6-15\% divergent for PV2), and (iii) Wild PVs [WPVs] (>15\% divergent) [3].

Originally, the strategy of the Global Polio Eradication Initiative in endemic countries mainly relied on the assessment of PV circulation through Acute Flaccid Paralysis (AFP) surveillance among children $<15$ years and extensive immunization with live-attenuated OPV. This strategy has led to the reduction of the incidence of WPV-associated poliomyelitis from an estimated 350,000 cases in 1988 to only 22 cases in 2017: 14 in Afghanistan and 8 in Pakistan [4]. At the same time, circulating VDPVs were reported only in two countries in 2017: 74 in Syria and 22 in Democratic Republic of Congo [5, 6].

PVs are transmitted by the feco-oral route through contaminated food, water and objects [7]. After ingestion, PV particles multiplies in the oropharynx and the intestine, and is excreted in stools for 4-6 weeks. The risk of PV infection and transmission has been shown to be

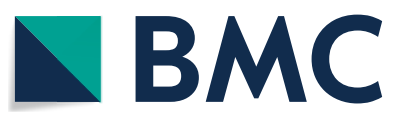

C The Author(s) 2019. This article is distributed under the terms of the Creative Commons Attribution 4.0 International License (http://creativecommons.org/licenses/by/4.0/), which permits unrestricted use, distribution, and reproduction in any medium, provided you give appropriate credit to the original author(s) and the source, provide a link to the Creative Commons license, and indicate if changes were made. The Creative Commons Public Domain Dedication waiver (http://creativecommons.org/ publicdomain/zero/1.0/) applies to the data made available in this article, unless otherwise stated. 
correlated with poor hygiene and sanitary conditions as well as high population density [8-10]. Most PV infections are generally asymptomatic but they can induce poliomyelitis in less than $1 \%$ of infected cases. Therefore, efficient AFP surveillance target only $1 \%$ of infected cases. Therefore the detection of PVs in environment samples is a useful supplement to monitor PV circulation in communities in absence of clinical presentations on which AFP surveillance relies.

Indigenous WPV transmission has originally been interrupted in Cameroon in 1999. However, WPV types 1 and 3 were repeatedly imported from endemic reservoirs into Cameroon from 2003 to 2014 [11]. In particular, isolation of highly evolved lineage of WPV type 1 associated to the 2013-2014 outbreak suggested potential gaps in the AFP surveillance system and vaccine coverage in Cameroon [11]. Moreover, circulating VDPVs, genetically linked to a previous outbreak in the neighboring Chad, caused a poliomyelitis outbreak in the Extreme Nord region of Cameroon in 2013 [12]. Appropriate responses with supplementary immunization activities were successful in stopping WPV and VDPV in Cameroon as from July 2014. Since July 2014, no clinical case of WPV and VDPV infections have been reported in Cameroon where national estimates of $P V$ vaccine coverage $\geq 83 \%$ have been documented from 2014 to 2017 [13]. In April 2016, Cameroon switched from trivalent OPV (tOPV; Sabin types 1, 2, and 3) to bivalent OPV (bOPV; Sabin types 1 and 3) after the introduction of one dose of inactivated PV vaccine type 2 in the routine immunization schedule. This switch was accompanied with the setup of environmental surveillance of PVs since January 2016.

This study aimed to (i) confirm the absence of circulating WPVs and VDPVs and (ii) investigate the detection rates and overtime distribution of the types of PVs recovered from urban sewage in Cameroon.

\section{Main text \\ Methods}

Between January 2016 and December 2017, 12 wastewater collection sites, including 8 in Yaounde and 4 in Douala, were selected on sewage drains covering populations at risk of PV transmission in Yaounde and Douala (Table 1). Collection sites were selected where wastewater flows were available from sites characterized by poor sanitation and high population density (Additional file 1: Figure S1). One liter of wastewater specimen was collected twice a week at each site on the due day. Specimens were transported in a reverse cold chain $\left(4-8{ }^{\circ} \mathrm{C}\right)$ to the Reference Laboratory for Poliomyelitis surveillance at the Centre Pasteur of Cameroon. Upon arrival, the $\mathrm{pH}$ of the specimens were eventually adjusted between 7 and 7.4 before virus concentration by the two-phase separation method using Dextran T40 and polyethylene glycol 6000 (PEG 6000) according to the standard protocol [14].

Water concentrates were analyzed according to the World Health Organization (WHO) guidelines for environmental surveillance of PV circulation [14] and the WHO polio laboratory manual [15]. These analyses comprised virus isolation, molecular differentiation of $\mathrm{PV}$ isolates, and sequencing of the VP1 capsid coding gene of Sabin 2 and other PV isolates identified as non-vaccine by molecular differentiation.

Two cell lines were used in this study: (i) L20B which are mouse fibroblast cells that have been transfected to express the PV-specific receptor CD155 and (ii) $\mathrm{RD}$ which are human rhabdomyosarcoma tumor cells expressing the majority of EV receptors including those for PVs. A volume of $500 \mu \mathrm{L}$ of each wastewater concentrate was inoculated into 5 flasks of L20B and 5 flasks of RD cells cultures maintained in Eagle's Minimum Essential Medium (Sigma-Aldrich) with 2\% decomplemented fetal calf serum at $36{ }^{\circ} \mathrm{C}$. Inoculated flasks were observed under an inverted objective microscope for 5 consecutive days to search for cytopathic effects (CPE). Isolates showing CPE only on RD but not on L20B cell cultures were classified as NPEVs. Those showing CPE on L20B cells cultures were considered as PVs.

Suspected PV isolates were typed by Intratypic differentiation (ITD) using real time RT-PCR (rRT-PCR) amplification with a combination of oligonucleotide sets as previously described $[15,16]$. This assay is able to identify the type of PV isolate and to discriminate between their wild and vaccine-related strains. Since type 2 OPV has been withdrawn from routine immunization, all Sabin 2 isolates identified by ITD were confirmed by the sequencing of their full-length VP1 capsid coding gene [17].

\section{Results}

A total of 517 sewage samples (325 from Yaounde and 192 from Douala) were collected from January 2016 to December 2017. EVs were detected in 33.5\% (173/517) of samples: 33.5\% (109/325) in Yaounde and 33.3\% (64/192) in Douala (Table 1, Additional file 1: Figure S1). Isolates were obtained from all studied sites in Douala in 2016 and 2017 whereas two sites in Yaounde (Nkoldongo and Aurore) showed no culture positive specimen in 2016 and 2017, respectively (Table 1). Remarkably, the Aurore site showed a virus isolation rate as low as $10.7 \%(3 / 28)$ while the isolation rates from other sites ranged from 22.2 to $47.7 \%$ and were comparable among the sites in both cities (Table 1). Both NPEV and PV detection rates were comparable between Yaounde and Douala $(\mathrm{P} \geq 0.2)$. 


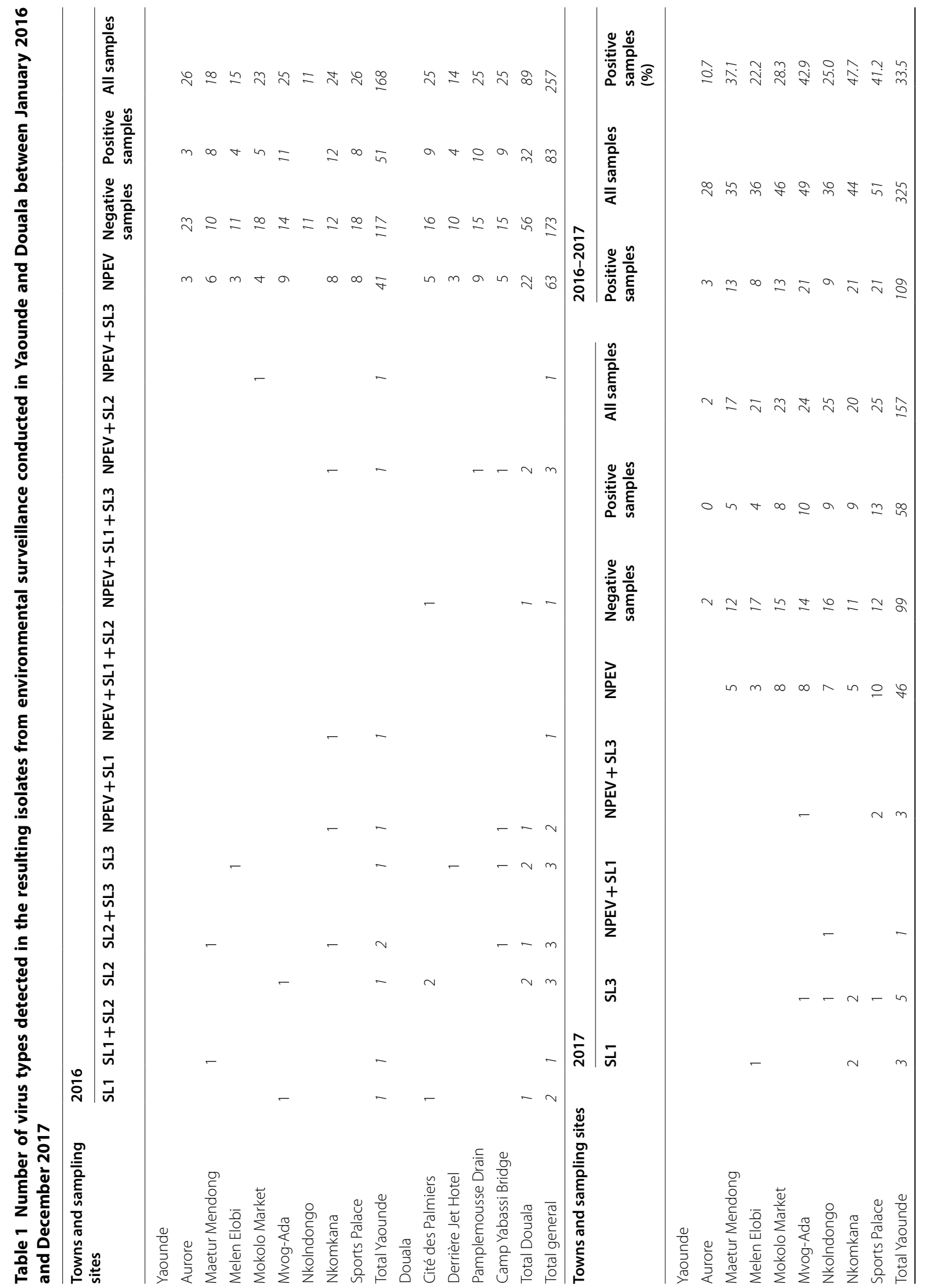




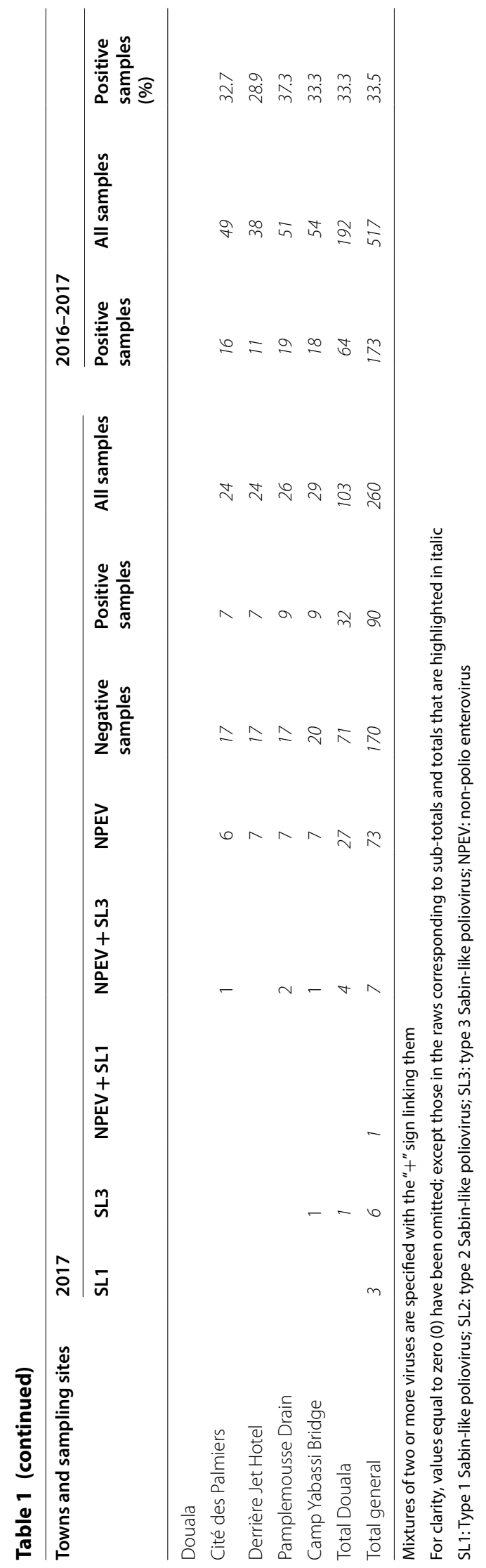




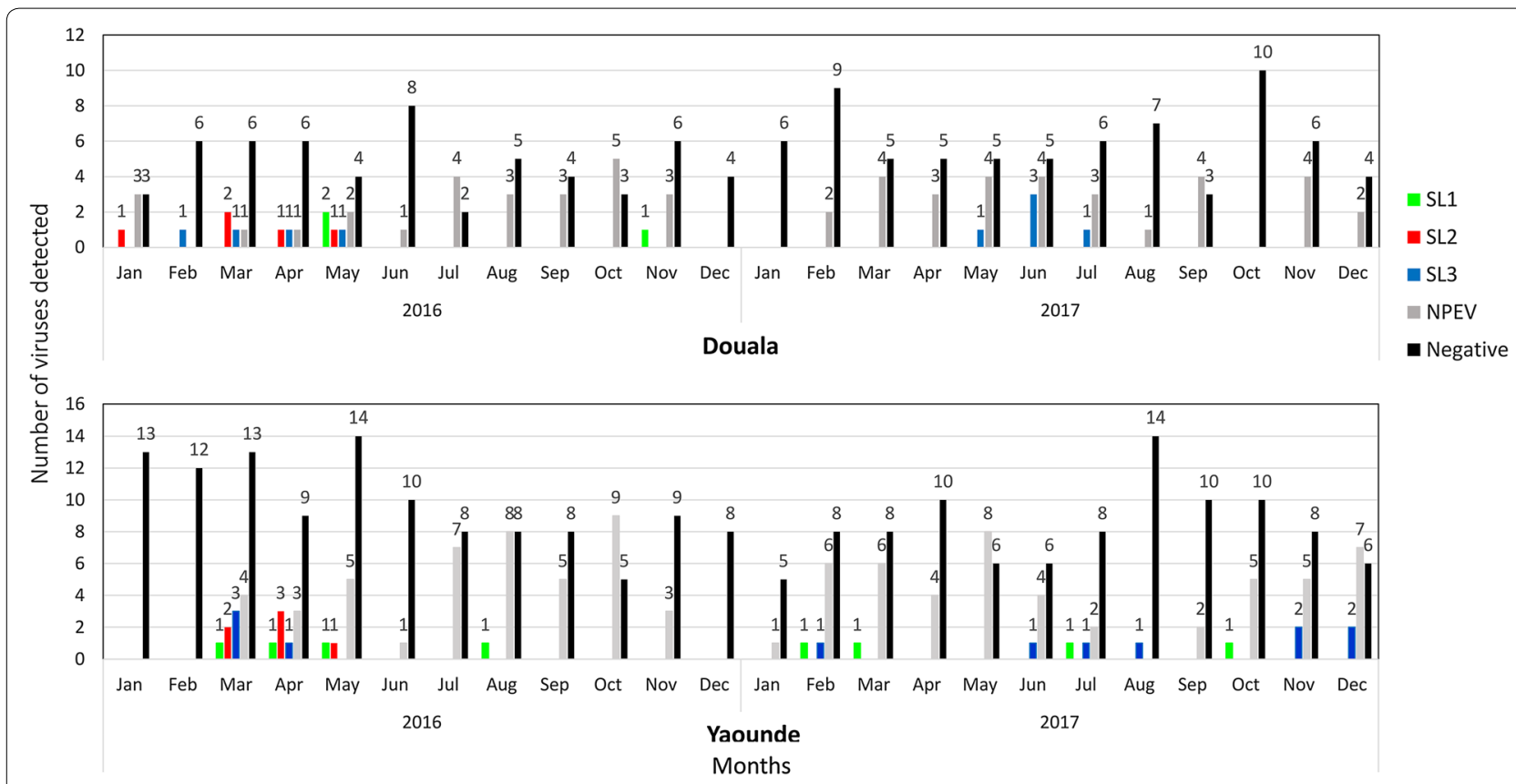

Fig. 1 Temporal pattern of the isolation of non-polio enteroviruses and vaccine polioviruses in the cities of Yaounde and Douala from January 2016 to December 2017. The number of individual virus types (SL1: Sabin type 1; SL2: Sabin type 2; SL3: Sabin type 3; NPEV: non-polio enterovirus) isolated and culture negative samples are indicated for each months

As expected, NPEVs represented the highest proportion of viruses detected irrespective of sample origin and month of collection (Fig. 1). NPEVs were detected in 136 (26.3\%) of samples including $87(26.7 \%)$ in Yaounde and $49(25.5 \%)$ in Douala. In contrast to NPEVs, PVs were detected only in $37(7.2 \%)$ sewage samples including 22 (6.8\%) in Yaounde and 15 (7.8\%) in Douala (Table 1).

Multiple virus types were simultaneously detected from some sample (Table 1). Considering individual isolates, a total of $43 \mathrm{PV}$ isolates (26 from Yaounde and 17 from Douala) were recovered from the 37 L20B-positive samples (Table 2). In contrast to NPEV that were consistently detected in all months in Yaounde and Douala during the study period, Sabin isolates were sporadically detected with comparable rates between Yaounde and Douala $(P \geq 0.8)$.

Although a pattern of seasonality could not be ruled out in this study, differences in the overtime distribution were noticeable in both cities. Sabin types 1 and 3 were isolated throughout the study period but their temporal distribution were apparently more dispersed in Yaounde than Douala (Fig. 1). Sabin type 2 displayed the same temporal distribution in both cities and their detection were limited between January and May 2016. Analysis of the VP1 sequence of these Sabin 2 isolates showed $\leq 2$ nucleotide difference compared to the original OPV type 2.

Interestingly, neither WPV nor VDPV were detected throughout the study period. These findings provide a substantial support to the observation from AFP surveillance which indicates that WPV and VDPV transmission has been successfully interrupted in Cameroon since 2014.

\section{Discussion}

The continuous isolation of NPEVs from sewage specimens from January 2016 to December 2017 confirms the extensive circulation of NPEVs as previously found among several populations in Cameroon [18-20]. The sporadic isolation of Sabin types 1 and 3 throughout the study period is consistent with the fact that Sabin 1 and Sabin 3 are still being used in routine immunization in Cameroon. In accordance with resent updates on the progress towards PV eradication [4, 21], no WPV isolate was identified during this study in Cameroon, thus suggesting the absence of silent WPV transmission in Yaoundé and Douala between January 2016 and December 2017.

Since the certification of the eradication of WPV type 2 in 2015, all countries that were using OPV switched from tOPV to bOPV from mid-April to mid-May 2016 [22]. Since then, the number of Sabin 2 isolated from both AFP cases and environmental specimens have progressively decline worldwide [23]. Accordingly, this study identified Sabin 2 isolates only during the first semester of 2016 (Fig. 1). The absence of WPV, VDPV as well as the early disappearance of Sabin 2, 1 month after the switch 
Table2 Number of samples analyzed and viruses detected in isolates resulting from environmental surveillance conducted in Yaounde and Douala between January 2016 and December 2017

\begin{tabular}{|c|c|c|c|c|c|c|c|c|c|c|c|c|c|c|}
\hline \multirow[t]{2}{*}{ Cities and sampling sites } & \multicolumn{6}{|c|}{2016} & \multicolumn{6}{|c|}{2017} & \multicolumn{2}{|c|}{ Total 2016-2017 } \\
\hline & $\mathbf{n}$ & SL1 & SL2 & SL3 & NPEV & All PVs & $\mathbf{n}$ & SL1 & SL2 & SL3 & NPEV & All PVs & $\mathbf{n}$ & All PVs (\%) \\
\hline \multicolumn{15}{|l|}{ Yaounde } \\
\hline Aurore & 26 & & & & 3 & 0 & 2 & & & & & 0 & 28 & $0(0.0)$ \\
\hline Maetur Mendong & 18 & 1 & 2 & 1 & 6 & 4 & 17 & & & & 5 & 0 & 35 & $4(11.4)$ \\
\hline Melen Elobi & 15 & & & 1 & 3 & 1 & 21 & 1 & & & 3 & 1 & 36 & $2(5.6)$ \\
\hline Mokolo Market & 23 & & & 1 & 5 & 1 & 23 & & & & 8 & 0 & 46 & $1(2.2)$ \\
\hline Mvog-Ada & 25 & 1 & 1 & & 9 & 2 & 24 & & & 2 & 9 & 2 & 49 & $4(8.2)$ \\
\hline Nkolndongo & 11 & & & & & 0 & 25 & 1 & & 1 & 8 & 2 & 36 & $2(5.6)$ \\
\hline Nkomkana & 24 & 2 & 3 & 1 & 11 & 6 & 20 & 2 & & 2 & 5 & 4 & 44 & $10(22.7)$ \\
\hline Sports Palace & 26 & & & & 8 & 0 & 25 & & & 3 & 12 & 3 & 51 & $3(5.9)$ \\
\hline Total Yaounde & 168 & 4 & 6 & 4 & 45 & 14 & 157 & 4 & 0 & 8 & 50 & 12 & 325 & $26(8.0)$ \\
\hline \multicolumn{15}{|l|}{ Douala } \\
\hline Cité des Palmiers & 25 & 2 & 2 & 1 & 6 & 5 & 24 & & & 1 & 7 & 1 & 49 & $6(12.2)$ \\
\hline Behind Jet Hotel & 14 & & & 1 & 3 & 1 & 24 & & & & 7 & 0 & 38 & $1(2.6)$ \\
\hline Pamplemousse Drain & 25 & & 1 & & 10 & 1 & 26 & & & 2 & 9 & 2 & 51 & $3(5.9)$ \\
\hline Camp Yabassi Bridge & 25 & 1 & 2 & 2 & 7 & 5 & 29 & & & 2 & 8 & 2 & 54 & $7(13.0)$ \\
\hline Total Douala & 89 & 3 & 5 & 4 & 26 & 12 & 103 & 0 & 0 & 5 & 31 & 5 & 192 & $17(8.9)$ \\
\hline Total general & 257 & 7 & 11 & 8 & 71 & 26 & 260 & 4 & 0 & 13 & 81 & 17 & 517 & $43(8.3)$ \\
\hline
\end{tabular}

For clarity, values equal to zero (0) have been omitted; except those in the raws corresponding to sub-totals and totals that are highlighted in italic

from tOPV to bOPV, are likely due to the high vaccine coverage of the target population in Cameroon. Indeed, national estimates of PV vaccine coverage was $\geq 83 \%$ between 2014 and 2017 in Cameroon compared to Nigeria where they have steadily been at $40 \%$ during the same period [13]. Transmission of WPV type 1 was originally thought to have also been interrupted in the neighboring Nigeria since July 2014 [24]. However, prolonged transmission of undetected WPV type 1 was recently reported in the Borno State of Nigeria [25]. Since 2016, type 2 circulating VDPV associated outbreaks have also been recently documented in multiple locations including Democratic Republic of Congo, Nigeria, Somalia, Pakistan and Syria; likely as result of the reduction of the population immunity against the PV type $2[5,24,26,27]$. Despite the absence of WPVs and circulating VDPVs in Cameroon since 2014, it remains essential to maintain high quality AFP surveillance supplemented by extended environmental surveillance in order to ensure rapid detection and control of VDPV emergence and WPV importations in polio-free countries.

\section{Limitations}

Although the present study is the first to investigate potential silent circulation of vaccine PVs, cVDPVs and WPVs in Cameroon, we focused only on the two biggest cities of Cameroon that are Yaounde and Douala. It would have been more interesting to include regions of
Cameroon that share borders with the northern states of Nigeria that are still considered as endemic for PVs. This limitation is already being addressed through the ongoing extension of environmental surveillance of PVs in Cameroon.

\section{Additional file}

Additional file 1: Figure S1. Map of Douala and Yaounde indicating selected sampling sites where sewage specimens were collected for environmental surveillance for enteroviruses. The cities of Yaounde and Douala are indicated by black circles on the map of Cameroon.

\section{Abbreviations}

AFP: Acute Flaccid Paralysis; CPE: cytopathic effects; EVs: enteroviruses; ITD: intratypic differentiation; NPEV: non polio enterovirus; OPV: oral poliovirus vaccine; PV: poliovirus; PV1, 2, 3: poliovirus types 1, 2, 3; VDPV: vaccine-derived poliovirus; WPV: wild poliovirus; WHO: World Health Organization.

\section{Authors' contributions}

MCEZ and BBPF, MDD, MNM, OMD and RN designed the study; MCEZ, BBPF, MDD and MNM coordinated field activities; DKN, SASM and MCEZ performed experiments and data analyses; MCEZ and RN administered the study; DKN and SASM wrote the first draft of the manuscript; all authors critically reviewed and approved the content of the final manuscript submitted. All authors read and approved the final manuscript.

\section{Author details}

${ }^{1}$ Virology Service, National Reference and Public Health Laboratory, Centre Pasteur of Cameroon, 451 Rue 2005, PO box 1274, Yaounde, Cameroon.

${ }^{2}$ World Health Organization, Country Office, PO box 155, Yaounde, Cameroon.

${ }^{3}$ Expanded Program on Immunization, Ministry of Public Health, Yaounde, 
Cameroon. ${ }^{4}$ The Polio Eradication Department, World Health Organization, Avenue Appia 20, 1211 Geneva 27, Switzerland.

\section{Acknowledgements}

We are grateful to field staffs of the ministry of health for their support during the collection of sewage specimens on the field.

\section{Competing interests}

The authors declare that they have no competing interests.

\section{Availability of data and materials \\ Not applicable.}

\section{Consent for publication}

Not applicable.

\section{Ethics approval and consent to participate}

Not required. Specimens were collected with the approval of the Cameroonian Ministry of Public Health within the framework of the environmental surveillance of polioviruses in Cameroon.

\section{Funding}

This study was supported by the World Health Organization through Technical Service Agreement (TSA) and the US Department of Health and Human Services, DHHS (Grant Number 6 DESP060001-01-01). The funders had no role in study design, data collection and analysis, decision to publish, or preparation of the manuscript.

\section{Publisher's Note}

Springer Nature remains neutral with regard to jurisdictional claims in published maps and institutional affiliations.

\section{Received: 11 March 2019 Accepted: 25 April 2019}

Published online: 02 May 2019

\section{References}

1. Nathanson N, Kew OM. From emergence to eradication: the epidemiology of poliomyelitis deconstructed. Am J Epidemiol. 2010;172:1213-29.

2. Jiang P, Faase JA, Toyoda H, Paul A, Wimmer E, Gorbalenya AE. Evidence for emergence of diverse polioviruses from C-cluster coxsackie A viruses and implications for global poliovirus eradication. Proc Natl Acad Sci U S A. 2007;104:9457-62.

3. Kew O, Pallansch M. Breaking the last chains of poliovirus transmission: progress and challenges in global polio eradication. Annu Rev Virol. 2018;5:427-51.

4. Khan F, Datta SD, Quddus A, Vertefeuille JF, Burns CC, Jorba J, Wassilak SGF. Progress toward polio eradication-Worldwide, January 2016-March 2018. MMWR Morb Mortal Wkly Rep. 2018;67:524-8.

5. Alleman MM, Chitale R, Burns CC, Iber J, Dybdahl-Sissoko N, Chen Q, Van Koko DR, Ewetola R, Riziki Y, Kavunga-Membo H, et al. Vaccine-derived poliovirus outbreaks and events - three provinces, Democratic Republic of the Congo, 2017. MMWR Morb Mortal Wkly Rep. 2018;67:300-5.

6. WHO: Polio Global Eradication Initiative; 2018. http://polioeradicatio n.org/where-we-work/polio-endemic-countries/. Accessed 2 Mar 2019.

7. Pallansch MA, Roos R. Enteroviruses: polioviruses, coxsackieviruses, echoviruses, and newer enteroviruses. In: Knipe DM, Howley PM, editors. Fields virology, vol. 1. 5th ed. Philadelphia: Lippincott Williams and Wilkins; 2007. p. 839-94.

8. Burns CC, Shaw J, Jorba J, Bukbuk D, Adu F, Gumede N, Pate MA, Abanida EA, Gasasira A, Iber J, et al. Multiple independent emergences of type 2 vaccine-derived polioviruses during a large outbreak in northern Nigeria. J Virol. 2013;87:4907-22.

9. Combelas N, Holmblat B, Joffret ML, Colbere-Garapin F, Delpeyroux F. Recombination between poliovirus and coxsackie $A$ viruses of species C: a model of viral genetic plasticity and emergence. Viruses. 2011;3:1460-84.
10. Kretsinger K, Gasasira A, Poy A, Porter KA, Everts J, Salla M, Brown KH, Wassilak SG, Nshimirimana D. Polio eradication in the World Health Organization African Region, 2008-2012. J Infect Dis. 2014;210:S23-39.

11. Endegue-Zanga MC, Sadeuh-Mba SA, Iber J, Burns CC, Moeletsi NG, Baba M, Bukbuk D, Delpeyroux F, Mengouo MN, Demanou M, et al. Importation and outbreak of wild polioviruses from 2000 to 2014 and interruption of transmission in Cameroon. J Clin Virol. 2016;79:18-24.

12. Endegue-Zanga MC, Sadeuh-Mba SA, Iber J, Burns C, Nimpa-Mengouo M, Demanou M, Vernet G, Etoa FX, Njouom R. Circulating vaccine-derived polioviruses in the Extreme North region of Cameroon. J Clin Virol. 2015;62:80-3.

13. WHO-UNICEF: WHO/UNICEF estimates of national immunization coverage; 2018. http://www.who.int/immunization/monitoring_surveillance/ routine/coverage/en/index4.html. Accessed 30 Sept 2018.

14. WHO. Guidelines for environmental surveillance of poliovirus circulation. WHO/V\&B/03.03. 2003rd ed. Geneva 27: World Health Organization; 2003.

15. WHO: World Health Organization. Polio laboratory manual, 4th edition. Geneva: The Organization. WHO/IVB/04.10. 2004.

16. Kilpatrick DR, Ching K, Iber J, Chen Q, Yang SJ, De L, Williams AJ, Mandelbaum M, Sun H, Oberste MS, Kew OM. Identification of vaccinederived polioviruses using dual-stage real-time RT-PCR. J Virol Methods. 2014;197:25-8.

17. Kilpatrick DR, Iber JC, Chen Q, Ching K, Yang SJ, De L, Mandelbaum MD, Emery B, Campagnoli R, Burns CC, Kew O. Poliovirus serotype-specific VP1 sequencing primers. J Virol Methods. 2011;174:128-30.

18. Ayukekbong J, Kabayiza JC, Lindh M, Nkuo-Akenji T, Tah F, Bergstrom T, Norder H. Shift of Enterovirus species among children in Cameroonidentification of a new enterovirus, EV-A1 19. J Clin Virol. 2013;58:227-32.

19. Ayukekbong JA, Andersson ME, Vansarla G, Tah F, Nkuo-Akenji T, Lindh M, Bergstrom T. Monitoring of seasonality of norovirus and other enteric viruses in Cameroon by real-time PCR: an exploratory study. Epidemiol Infect. 2014;142:1393-402.

20. Sadeuh-Mba SA, Bessaud M, Massenet D, Joffret ML, Endegue MC, Njouom R, Reynes JM, Rousset D, Delpeyroux F. High frequency and diversity of species $C$ enteroviruses in Cameroon and neighboring countries. J Clin Microbiol. 2013;51:759-70.

21. Fournier-Caruana J, Previsani N, Singh H, Boualam L, Swan J, Llewellyn A, Sutter RW, Zaffran M. Progress toward poliovirus containment implementation-worldwide, 2017-2018. MMWR Morb Mortal Wkly Rep. 2018;67:992-5.

22. Hampton LM, Farrell M, Ramirez-Gonzalez A, Menning L, Shendale S, Lewis I, Rubin J, Garon J, Harris J, Hyde T, et al. Cessation of trivalent oral poliovirus vaccine and introduction of inactivated poliovirus vaccineworldwide, 2016. MMWR Morb Mortal Wkly Rep. 2016;65:934-8.

23. Diop OM, Asghar H, Gavrilin E, Moeletsi NG, Benito GR, Paladin F, Pattamadilok S, Zhang Y, Goel A, Quddus A. Virologic monitoring of poliovirus type 2 after oral poliovirus vaccine type 2 withdrawal in April 2016worldwide, 2016-2017. MMWR Morb Mortal Wkly Rep. 2017;66:538-42.

24. Etsano A, Damisa E, Shuaib F, Nganda GW, Enemaku O, Usman S, Adeniji A, Jorba J, Iber J, Ohuabunwo C, et al. Environmental isolation of circulating vaccine-derived poliovirus after interruption of wild poliovirus transmission-Nigeria, 2016. MMWR Morb Mortal Wkly Rep. 2016;65:770-3.

25. Nnadi C, Damisa E, Esapa L, Braka F, Waziri N, Siddique A, Jorba J, Nganda GW, Ohuabunwo C, Bolu O, et al. Continued endemic wild poliovirus transmission in security-compromised areas-Nigeria, 2016. MMWR Morb Mortal Wkly Rep. 2017;66:190-3.

26. Eboh VA, Makam JK, Chitale RA, Mbaeyi C, Jorba J, Ehrhardt D, Durry E, Gardner T, Mohamed K, Kamugisha C, et al. Notes from the field: widespread transmission of circulating vaccine-derived poliovirus identified by environmental surveillance and immunization response-Horn of Africa, 2017-2018. MMWR Morb Mortal Wkly Rep. 2018;67:787-9.

27. Nanteza MB, Bakamutumaho B, Kisakye A, Namuwulya P, Bukenya H, Katushabe E, Bwogi J, Byabamazima CR, Williams R, Gumede N. The detection of 3 ambiguous type 2 vaccine-derived polioviruses (VDPV2s) in Uganda. Virol J. 2018;15:77. 